\title{
Pharmacokinetic Differences of Three Aconitum Alkaloids from Aconiti lateralis Radix Praeparata and Compatibility with Pinellia in Rats
}

\author{
Wu Jian ${ }^{1,2 *}$, Li Wen-lan ${ }^{1,2}$, Xu Ying ${ }^{1,2}$, Liu Ying-jie ${ }^{1,2}$, Liu Ri-jia1,2, Yu Miao ${ }^{1,2}$ \\ ${ }^{1}$ Department of Pharmacy, Harbin University of Commerce, Harbin 150076, CHINA. \\ ${ }^{2}$ Engineering Research Center of Natural Antineoplastic Drugs, Ministry of Education, Harbin 150076, CHINA.
}

\begin{abstract}
Aim: This study aims to develop a highly sensitive and specific UPLC-MS/MS method to explore the pharmacokinetic properties of three representative active alkaloids (benzoylhypaconine, benzoylmesaconine, benzoylaconine) after administration of extracts of Aconiti lateralis Radix Praeparata and compatibility with Pinellia, compare the influence of Pinellia on pharmacokinetic characterization of three aconitum alkaloids. Methods: Chromatographic separation were performed on a $\mathrm{C}_{18}$ column under the Multiple reaction monitoring (MRM) in the positive Electrospray ionization (ESI) mode. The pharmacokinetic parameters were evaluated by software DAS 3. 0. Results: The specificity, precision and accuracy, matrix effect and extraction recovery rate and stability all meet the requirements. The validation of the method was achieved in accordance to the FDA guidelines. After oral administration of the extract, similar tendency was found in the mean concentration of the three analytes in the curves, illustrating that three analytes exhibited a similar absorption and metabolic route. Compared the pharmacokinetic characteristics, the $A \cup C_{0-t^{\prime}} A \cup C_{0-\infty}$ and $C_{\max }$ values of $\mathrm{BMA}$ and $\mathrm{BHA}$ in Fuzi group were lower than those in Fuzi-Banxia group. Conclusion: This finding indicated the significant statistical decrease $(p<0.05)$ in the absorption of these components. After Banxia in combination with Fuzi, the results showed that faster absorption and higher exposure of BMA and BHA in rat plasma.
\end{abstract}

Key words: Pharmacokinetic, Aconiti lateralis Radix, Pinellia, Aconitum alkaloids, UPLC-MS/MS.

\section{INTRODUCTION}

Most herbal medicines were prescribed in combination based on the theory of traditional Chinese herbal medicine to obtain synergistic effects or diminish the possible adverse reaction, ${ }^{1}$ but meanwhile, there also have a special rule for TCM named Eighteen Incompatible Pairs. Incompatible pairs means two herbs are not suitable for clinical application together, otherwise there will be detrimental to therapeutic efficacy, causing toxicity to the body. ${ }^{2}$ As an empirical summary obtained from long-term TCM clinical practice, the incompatibility theory is closely related to safe medication and has gained increasing concerns for modernization research of TCMs. ${ }^{3}$ As recorded in Chinese Pharmacopeia, co-administration of Pinellia Banxia) and Aconiti lateralis Radix Praeparata (Fuzi) was prohibited. ${ }^{4}$ It has always been disputed, because there are many traditional Chinese herbal prescriptions including incompatible pairs, such as Wufu Jingfang, containing Aconiti lateralis Radix Praeparata and Pinellia.

In order to resolve the mystery of the ancient rule of TCM incompatibility, scientific experiments based on chemistry and pharmacology were carried out recently., ${ }^{5,6}$ Nevertheless, these methods are not sensitive enough for trace detection in a pharmacokinetic study.
Submission Date: 31-07-2018; Revision Date: 23-11-2018; Accepted Date: 29-12-2018

DOI: 10.5530/ijper.53.2.31 Correspondence: WU Jian,

Research Center on Life Sciences and Environmental Sciences, Harbin University of Commerce, Harbin 150076, CHINA.

Phone: +0086 0451 84844242

E-mail:wujian071@163.com

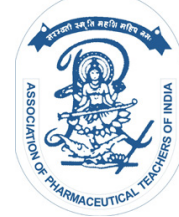

www.ijper.org 
The powerful and reliable UPLC-MS/MS method and Multiple reaction monitoring technology has been widely used in pharmacokinetic studies because it can provide high sensitivity for quantification. The use of MRM technology improves the analytical selectivity and sensitivity and eliminate obvious endogenous interference from plasma. Fuzi, derived from the dried daughter root of Aconitum carmichaelii, three monoester alkaloids (MDAs) were considered to be the effective as well as the toxic components. In particular, the monoesterditerpene alkaloids have a narrow therapeutic index. ${ }^{7}$ As a highly toxic drug, there is little information available on the pharmacokinetic studies of the alkaloid constituents after compatibility with Banxia. In this study, a rapid, sensitive and robust UPLC-MS/MS method for simultaneous determination of benzoylaconine, benzoylmesaconine and benzoylhypaconine in plasma was developed and validated. Pharmacokinetic study was performed to explore the in vivo differences of the three alkaloids after oral administration of Fuzi-Banxia and each single herb extracts.

\section{MATERIALS AND METHODS}

\section{Chemical and reagents}

Aconiti lateralis Radix Praeparata and Pinellia, were purchased from the shan ke shu Medical Material Company (Harbin, China) and authenticated by Professor Yu-bin JI (Harbin University of Commerce, Harbin, China). The voucher specimens were deposited in the laboratory of Research Center on Life Sciences and Environmental Sciences (Harbin University of Commerce). Benzoylaconine, benzoylmesaconine, benzoylhypaconine and pentoxifylline (internal standard, IS) were all purchased from the National Institutes for Food and Drug Control (Beijing, China); Methanol, acetonitrile and formic acid (HPLC grade) was purchased from Sigma (St. Louis, MO, USA); Deionized water was purified on a Milli-Q system (Millipore, Milford, MA, USA).

\section{Animals}

Male Sprague-Dawley rats $(200 \pm 20$ g) were supplied by the Laboratory Animal Center of Harbin Medical University (Harbin, China). Eighteen healthy male SD rats were randomly divided into three groups $(n=6)$ and were given Fuzi, Banxia or Fuzi-Banxia extracts by gastric perfusion, respectively $(20 \mathrm{~g} / \mathrm{kg}$, the single herb/ body weight). The study was approved by Animal Ethic Committee of the Harbin University of Commerce (No. EA_20160107) and the experiments with rats were in full compliance with the European Communities
Council Directive of 24 November 1986 (86/609/EEC) and with the Guidelines laid down by the NIH in the US.

\section{Preparation of Fuzi-Banxia extracts}

Fuzi, Banxia and Fuzi-Banxia herb pair (at the ratio of 1:1) were weighed respectively, soaked in water (10 folds) for $1 \mathrm{~h}$, then refluxed for $0.5 \mathrm{~h}, 3$ times altogether. After filtering with 3 layers of gauze, the filtrates were merged and concentrated to a concentration of $1.0 \mathrm{mg} / \mathrm{mL}$ (equivalent to the single herb). The aqueous extracts were stored at $4^{\circ} \mathrm{C}$ until administration.

\section{Preparation of standard solutions, calibration and quality control (QC) samples}

Benzoylaconine, benzoylmesaconine, benzoylhypaconine were accurately weighed and dissolved in a watermethanol (50:50, v/v) solution. Working solutions were obtained by serially diluting the stock solutions in water-methanol $(50: 50, \mathrm{v} / \mathrm{v})$ solution to produce the final concentrations of $0.5-500 \mathrm{ng} / \mathrm{mL}$ for benzoylmesaconine, $0.25-250 \mathrm{ng} / \mathrm{mL}$ for benzoylhypaconine and $0.25-250 \mathrm{ng} / \mathrm{mL}$ for benzoylaconine. The concentration of IS was $25 \mathrm{ng} / \mathrm{mL}$ in methanol. Before use, all of the solutions were stored at $4^{\circ} \mathrm{C}$. Calibration samples were prepared by spiking $5 \mu \mathrm{L}$ of working solutions at the corresponding concentrations into $45 \mu \mathrm{L}$ of blank rat plasma. The Lower Limit of Quantification (LLOQ), low, medium and high quality control (QC) samples were prepared in the same way. All solutions were stored in a refrigerator $\left(-40^{\circ} \mathrm{C}\right)$ until analysis.

\section{Instrumentation and LC-MS/MS analytical conditions}

An Agilent 1290 series UPLC system (Agilent Technologies, Santa Clara, CA, USA) consisted of a binary pump, an online degasser, an auto plate sampler and a thermostatically-controlled compartment. Separation of analytes was performed on a Waters Acquilty UPLC $\mathrm{C}_{18}$ column $(100 \mathrm{~mm} \times 2.1 \mathrm{~mm}, 1.7 \mu \mathrm{M}$, Waters, Milford, MA, USA). The mobile phase was composed of $0.1 \%$ formic acid (A) and acetonitrile (B) using a gradient elution of $95-80 \% \mathrm{~A}$ at $0-2 \mathrm{~min}, 80-70 \% \mathrm{~A}$ at $2-3 \mathrm{~min}, 70-50 \% \mathrm{~A}$ at $3-4 \mathrm{~min}, 50 \% \mathrm{~A}$ at $4-4.4 \mathrm{~min}$ and the re-equilibration time of gradient elution was $0.6 \mathrm{~min}$. The injection volume was $5 \mu \mathrm{L}$ and the flow rate was $0.35 \mathrm{~mL} / \mathrm{min}$. The MRM transitions included $\mathrm{m} / \mathrm{z} \quad 590.3 \rightarrow 105.1$ for benzoylmesaconine, with a collision energy of $49 \mathrm{eV} ; \mathrm{m} / \mathrm{z} \quad 574.3 \rightarrow 105.1$ for benzoylhypaconine, with a collision energy of $50 \mathrm{eV}$; $\mathrm{m} / \mathrm{z} 604.1 \rightarrow 105.1$ for benzoylaconine, with a collision energy of $49 \mathrm{eV} ; \mathrm{m} / \mathrm{z} 278.3 \rightarrow 180.1$ for pentoxifylline, with a collision energy of $49 \mathrm{eV}$. Other parameters were as follows: carrier gas $\left(\mathrm{N}_{2}\right)$ flow rate, $11.0 \mathrm{~L} / \mathrm{min}$. 


\section{Sample preparation}

Blood samples $(100 \mu \mathrm{L})$ were collected from the suborbital venous lexus at $5,10,15,30,45,60,90,120$, 240, 360, 480,720 and $1440 \mathrm{~min}$ after administration. Collected blood samples were placed into heparinized tubes and centrifuged at $4500 \mathrm{r} / \mathrm{min}$ for $15 \mathrm{~min}$ and the plasma was stored at $-40^{\circ} \mathrm{C}$ before analysis. $50 \mu \mathrm{L}$ plasma sample and $200 \mu \mathrm{L}$ IS $(25.0 \mathrm{ng} / \mathrm{mL})$ vortexed for $3 \mathrm{~min}$. The mixture was centrifuged at $13500 \mathrm{r} / \mathrm{min}$ for $15 \mathrm{~min}$. The organic layer was separated, transferred and dried under nitrogen at $40^{\circ} \mathrm{C}$. Mobile phase $(50 \mu \mathrm{L})$ was applied to reconstitute the residue and then the mixture was vortexed for $3 \mathrm{~min}$, followed by centrifugation at $13500 \mathrm{r} / \mathrm{min}$ for $5 \mathrm{~min}$. For analysis, $5 \mu \mathrm{L}$ of the supernatant was injected.

\section{Method validation}

Method validation was performed to evaluate selectivity, sensitivity, linearity, accuracy and precision, recovery, matrix effect and stability according to the Guidance for Industry-Bioanalytical Method Validation recommended by the US Food and Drug Administration (FDA, 2014).

\section{Selectivity}

The selectivity of the method was evaluated by analyzing blank plasma samples from six different rats, plasma samples spiked with benzoylaconine, benzoylmesaconine, benzoylhypaconine and IS were analyzed and compared to assess the selectivity of the method, as shown in figure 1.

\section{Linearity and low limits of quantification (LLOQ)}

A $1 / x^{2}$ weighted least square linear regression model was used to construct calibration curves by plotting the peak-area ratios of each analyte to IS versus plasma concentrations. LLOQ was determined based on the two criteria: (1) the analyte response at the LLOQ would be at least 10 times that of the blank; and (2) the analyte peak would be discrete and reproducible with a precision within $20 \%$ and accuracy of $80 \%-120 \%$ (Table 1 ).

\section{Precision and accuracy}

The inter-day precision and accuracy were determined on three consecutive days and the intra-day precision was evaluated on the same day by determination of QC samples. Analysis $(n=6)$ of QC samples at different concentrations (low, middle and high) and one calibration curve was included in each run. Relative standard deviation (RSD) was used to express the precision and relative error (RE) was applied to express the accuracy.

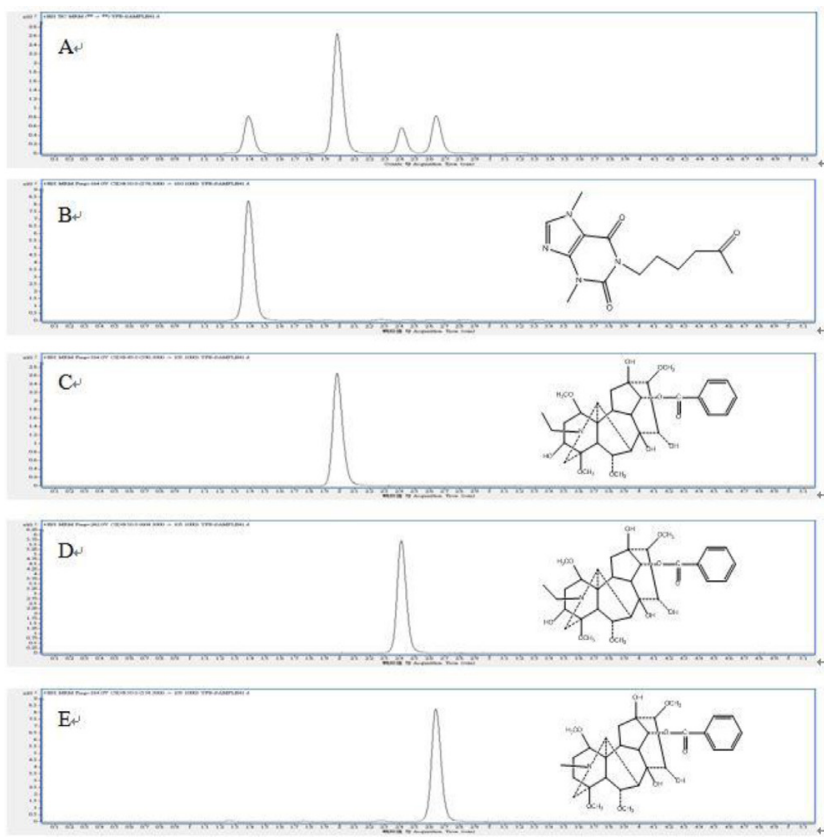

Figure 1: A Chromatograms of blank plasma samples with benzoylmesaconine $T_{r}=2.05 \mathrm{~min}$, benzoylaconine $T_{r}=2.42 \mathrm{~min}$, benzoylhypaconine $T_{r}=2.65 \mathrm{~min}$, pentoxifylline (IS) $T_{r}=1.39 \mathrm{~min} ; B$. Extract chromatograms of plasma sample of $30 \mathrm{~min}$ after administration of extracts pentoxifylline (IS) $T_{r}=1.39 \mathrm{~min}, \mathrm{C}$. Extract chromatograms of plasma sample of $30 \mathrm{~min}$ after administration of extracts benzoylmesaconine $T_{r}=2.05 \mathrm{~min}, \mathrm{D}$. Extract chromatograms of plasma sample of $30 \mathrm{~min}$ after administration of extracts benzoylaconine $T_{r}=2.42 \mathrm{~min}$, $E$. Extract chromatograms of plasma sample of 30 min after administration of extracts benzoylhypaconine $\mathrm{T}_{\mathrm{r}}=2.65 \mathrm{~min}$

\section{Extraction recovery and the matrix effect}

Extraction recovery experiments were performed by comparing the mean peak area of extracted samples (spiked before extraction) to the peak area of unextracted samples (QC working solutions spiked in extracted plasma) at LQC, MQC and HQC levels. The matrix effect was assessed by comparing the peak area obtained from an amount of the analyte added in extracted blank plasma to the peak area obtained from equal concentration of the analyte in solvent at low, medium and high concentrations in Table 2 .

\section{Stability}

QC samples at three concentrations under different sample storage conditions and processing procedures. Short-term stability ( $4 \mathrm{~h}$ at room temperature), longterm stability $\left(15 \mathrm{~d}\right.$ at $\left.-40^{\circ} \mathrm{C}\right)$, freeze-thaw stability (three cycles, from $-40^{\circ} \mathrm{C}$ to room temperature as a cycle) and post-preparation stability $\left(12 \mathrm{~h}\right.$ at $\left.4^{\circ} \mathrm{C}\right)$ were determined and summarized in Table 3. 


\begin{tabular}{|c|c|c|c|c|}
\hline Analytes & Linear range (ng mL-1) & Regression equation & $\mathbf{R}^{2}$ & LLOQ \\
\hline benzoylmesaconine & $0.500 \sim 500$ & $Y=0.00124 x+0.0143$ & 0.9961 & 0.500 \\
\hline benzoylaconine & $0.250 \sim 250$ & $Y=0.00116 x-0.00104$ & 0.9968 & 0.250 \\
\hline benzoylhypaconine & $0.250 \sim 250$ & $Y=0.00454 x+0.00614$ & 0.9959 & 0.250 \\
\hline
\end{tabular}

\begin{tabular}{|c|c|c|c|c|c|c|}
\hline \multicolumn{3}{|c|}{ Table 2: Precision and accuracy for the determination of three analytes in rat plasma (n = 6). } \\
\hline \multirow{2}{*}{ analytes } & $\begin{array}{c}\text { concentration } \\
(\mathbf{n g} / \mathbf{m L})\end{array}$ & $\begin{array}{c}\text { Inter-day } \\
\text { RSD (\%) }\end{array}$ & $\begin{array}{c}\text { Intra-day } \\
\text { RSD (\%) }\end{array}$ & $\begin{array}{c}\text { Precision } \\
\text { (\%) }\end{array}$ & $\begin{array}{c}\text { Extraction } \\
\text { recovery } \\
(\%)\end{array}$ & $\begin{array}{c}\text { Matrix effect } \\
(\%)\end{array}$ \\
\hline \multirow{3}{*}{ benzoylmesaconine } & 1.00 & 5.8 & 3.8 & $96.5 \%$ & $101.3 \pm 2.9$ & $102.9 \pm 4.1$ \\
\cline { 2 - 7 } & 20.0 & 6.5 & 5.4 & $100.3 \%$ & $98.5 \pm 4.2$ & $102.5 \pm 2.7$ \\
\cline { 2 - 7 } & 400 & 4.5 & 4.7 & $97.1 \%$ & $95.6 \pm 3.8$ & $97.4 \pm 5.4$ \\
\hline \multirow{3}{*}{ benzoylaconine } & 0.500 & 6.2 & 5.2 & $100.2 \%$ & $97.8 \pm 3.9$ & $103.8 \pm 2.4$ \\
\cline { 2 - 7 } & 10.0 & 7.4 & 5.1 & $97.8 \%$ & $96.5 \pm 4.1$ & $98.4 \pm 4.5$ \\
\hline \multirow{2}{*}{ benzoylhypaconine } & 200 & 5.5 & 4.3 & $99.6 \%$ & $97.1 \pm 2.6$ & $97.1 \pm 3.2$ \\
\cline { 2 - 7 } & 0.500 & 5.3 & 6.4 & $102.4 \%$ & $102.1 \pm 3.2$ & $102.4 \pm 4.1$ \\
\cline { 2 - 7 } & 10.0 & 7.2 & 4.9 & $103.6 \%$ & $96.4 \pm 2.7$ & $101.4 \pm 2.9$ \\
\hline
\end{tabular}

\begin{tabular}{|c|c|c|c|c|c|c|c|c|c|}
\hline \multirow[b]{2}{*}{ Analytes } & \multirow{2}{*}{$\begin{array}{c}\text { Concentration } \\
\mathrm{ng} / \mathrm{mL}\end{array}$} & \multicolumn{2}{|c|}{$25^{\circ} \mathrm{C}$ for $4 \mathrm{~h}$} & \multicolumn{2}{|c|}{$-40^{\circ} \mathrm{C}$ for 15 days } & \multicolumn{2}{|c|}{ freeze-thaw cycles } & \multicolumn{2}{|c|}{$4^{\circ} \mathrm{C}$ for $24 \mathrm{~h}$} \\
\hline & & $\begin{array}{l}\text { RSD } \\
(\%)\end{array}$ & $\begin{array}{c}\text { Remaining } \\
(\%)\end{array}$ & $\begin{array}{l}\text { RSD } \\
(\%)\end{array}$ & $\begin{array}{c}\text { Remaining } \\
(\%)\end{array}$ & $\begin{array}{l}\text { RSD } \\
(\%)\end{array}$ & $\begin{array}{c}\text { Remaining } \\
(\%)\end{array}$ & $\begin{array}{c}\text { RSD } \\
(\%)\end{array}$ & $\begin{array}{c}\text { Remaining } \\
(\%)\end{array}$ \\
\hline \multirow{3}{*}{ benzoylmesaconine } & 1.00 & 8.6 & 100.5 & 8.4 & 101.2 & 7.2 & 95.4 & 6.7 & 102.3 \\
\hline & 20.0 & 5.3 & 101.6 & 7.6 & 103.5 & 7.8 & 100.1 & 8.0 & 98.9 \\
\hline & 400 & 5.7 & 101.2 & 5.4 & 104.5 & 5.6 & 104.8 & 7.6 & 100.9 \\
\hline \multirow{3}{*}{ benzoylaconine } & 0.500 & 8.2 & 101.9 & 6.2 & 104.7 & 8.4 & 97.4 & 5.8 & 101.2 \\
\hline & 10.0 & 5.4 & 102.6 & 5.9 & 102.6 & 5.6 & 101.4 & 6.1 & 103.2 \\
\hline & 200 & 4.6 & 103.7 & 4.2 & 102.1 & 6.7 & 105.6 & 6.4 & 102.5 \\
\hline \multirow{3}{*}{ benzoylhypaconine } & 0.500 & 8.7 & 104.6 & 7.3 & 103.2 & 5.2 & 98.9 & 6.8 & 103.0 \\
\hline & 10.0 & 5.2 & 103.4 & 6.7 & 104.8 & 7.4 & 101.4 & 7.6 & 97.5 \\
\hline & 200 & 5.4 & 102.3 & 4.1 & 102.3 & 5.4 & 103.6 & 7.9 & 102.6 \\
\hline
\end{tabular}

Table 4: Pharmacokinetic parameters of alkaloids after the oral administration of Wutou decoction in rats $(n=6)$.

\begin{tabular}{|c|c|c|c|c|c|c|c|}
\hline \multicolumn{2}{|c|}{ analytes } & $\begin{array}{l}T_{1 / 22} \\
(\min )\end{array}$ & $\underset{(n g / m L)}{C_{\max }}$ & $\underset{\text { (min) }}{T_{\max }}$ & $\begin{array}{c}\text { AUC }_{0-\mathrm{t}} \\
\left.\text { (ng }{ }^{*} \mathrm{~min} / \mathrm{ml}\right)\end{array}$ & $\begin{array}{c}\text { AUC }_{0-\infty} \\
\left(\mathrm{ng}^{*} \mathrm{~min} / \mathrm{ml}\right)\end{array}$ & $\begin{array}{l}\text { MRT }_{0-t} \\
(\min )\end{array}$ \\
\hline \multirow{2}{*}{ benzoylmesaconine } & Fuzil & $661 \pm 75$ & $43.2 \pm 15.6$ & $30(30,45)$ & $5019 \pm 1023$ & $5954 \pm 803$ & $564 \pm 31$ \\
\hline & Fuzi-Banxia & $654 \pm 42$ & $86.2 \pm 7.14^{*}$ & $30(30,45)$ & $9624 \pm 235^{\star}$ & $10717 \pm 426^{*}$ & $537 \pm 25$ \\
\hline \multirow{2}{*}{ benzoylaconine } & Fuzil & $493 \pm 36$ & $25.4 \pm 14.3$ & $30(30,45)$ & $3046 \pm 386$ & $4059 \pm 326$ & $304 \pm 38$ \\
\hline & Fuzi-Banxia & $474 \pm 38$ & $23.4 \pm 13.2$ & $30(30,45)$ & $2945 \pm 264$ & $3859 \pm 214$ & $345 \pm 26$ \\
\hline \multirow{2}{*}{ benzoylhypaconine } & Fuzil & $452 \pm 47$ & $29.8 \pm 11.4$ & $30(30,45)$ & $3319 \pm 156$ & $4225 \pm 168$ & $372 \pm 31$ \\
\hline & Fuzi-Banxia & $436 \pm 39$ & $66.9 \pm 6.51^{*}$ & $30(30,45)$ & $7045 \pm 39^{*}$ & $8025 \pm 134^{*}$ & $395 \pm 38$ \\
\hline
\end{tabular}

*Significantly from oral administration of extract in rats $(p<0.05)$ 


\section{Pharmacokinetic study}

Plasma concentration-time curves were plotted and pharmacokinetic parameters were calculated by using the DAS 3.0 pharmacokinetic analysis software. All the data were presented as mean \pm standard deviation (SD). Analysis of the data was executed by non-compartmental model and statistical moment calculation, from which pharmacokinetic parameters were obtained. The Student's test was used to test significant differences between Fuzi group and Fuzi-Banxia group and $p<0.05$ was considered as statistically significant.

\section{RESULTS AND DISCUSSION}

\section{LC-MS/MS condition}

Positive ESI ion modes were employed to achieve the mass spectra of benzoylaconine, benzoylmesaconine, benzoylhypaconine and IS. The responses for each compound were higher in positive mode. Precursor ions and product ions were monitored by MRM to enhance selectivity and lower interference. The capillary and cone voltages as well as the source temperature and desolvation gas temperature/flow were selected to optimize the specificity and sensitivity of protonated analyte molecules. To obtain the maximum response of the analyte fragmentation, as well as IS, the collision energy of

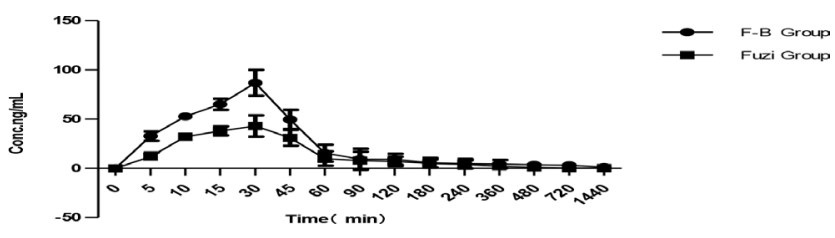

Figure 2: Plasma concentration-time curves of benzoylmesaconine after administration of extracts.

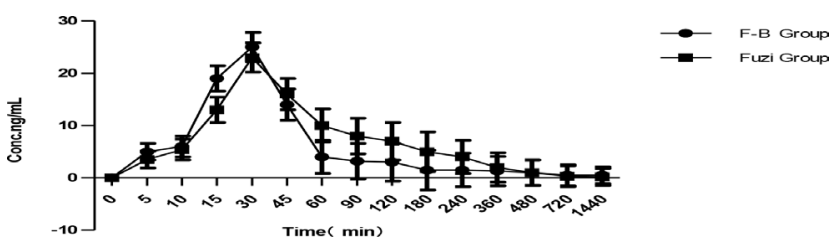

Figure 3: Plasma concentration-time curves of benzoylaconine after administration of extracts.

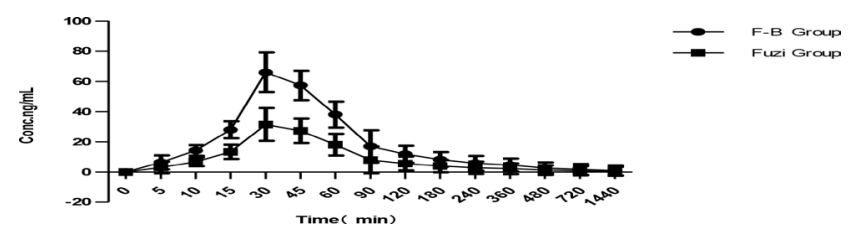

Figure 4: Plasma concentration-time curves of benzoylhypaconine after administration of extracts.
Collision-Induced Decomposition (CID) was optimized.

\section{Pharmacokinetic study}

The validated assay was applied for comparative pharmacokinetic study of the three alkaloids in Fuzi group and Fuzi-Banxia group after oral administration of extract. The mean plasma concentration-time curves (mean $\pm \mathrm{SD}$ ) of the three analytes are presented in Figure 2-4 and the corresponding pharmacokinetic parameters are summarized in Table 4 . After oral administration of the extract, similar tendency was found in the mean concentration of the three analytes in the curves, illustrating that three analytes exhibited a similar absorption and metabolic route. Compared the pharmacokinetic characteristics, the $\mathrm{AUCO}_{-}, \mathrm{AUCO}_{-}$and $\mathrm{C}_{\max }$ values of BMA and BHA in Fuzi group were lower than those in Fuzi-Banxia group. This finding indicated the significant statistical decrease $(p<0.05)$ in the absorption of these components. After Banxia in combination with Fuzi, the results showed that faster absorption and higher exposure of BMA and $\mathrm{BHA}$ in rat plasma.

\section{CONCLUSION}

In this study, a rapid, sensitive and specific UPLC-MS/MS method was established and applied to a comparative pharmacokinetic study of three aconitine-type alkaloids after oral administration of Fuzi, Banxia and Fuzi-Banxia extracts. Combined administration of Fuzi and Banxia accelerated the absorption of BMA and BHA, this comparative pharmacokinetic study would be helpful for improving clinical therapeutic efficacy and further pharmacological studies of Fuzi and Banxia and their herb-herb interaction.

\section{ACKNOWLEDGEMENT}

This work was financially supported by the Youth Innovative Talent Project of Harbin University of Commerce (2016QN055).

\section{CONFLCIT OF INTEREST}

The authors declare no conflict of interest.

\section{ABBREVIATIONS}

BMA: Benzoylmesaconine; BHA: Benzoylhypaconine; BAC: Benzoylaconine; UPLC-MS/MS: Ultra-High Performance Liquid Chromatography-Tandem Mass Spectrometry. 


\section{REFERENCES}

1. Buriani A, Garcia-Bermejo ML, Bosisio E. Omictechniques in systems biology approaches to traditional Chinese medicine research: present and future. J Ethnopharmacol. 2012;140(3):535-44.

2. Chen S, Zhao G. Application of compatibility of Radix Aconiti lateralis Praeparata with its incompatible medicaments Pinelliae Rhizoma, Trichosanthis Fructus and Fritillariae Cirrhosae bulbus. Mode Trad Chin Med. 2007;27:60-1.

3. Chinese Pharmacopoeia Commission, Pharmacopoeia of the People's Republic of China, China Medical Science Press, Beijing, China. 2015;191-292.
4. Wang YG, Ma ZC, Liang QD, Liu M, Lu BB, Tan HL, et al. Study on the eighteen incompatible pairs based on cytochrome P450. Tradit Chin Med Mater Med. 2011;13(1):36-40.

5. Singhuber J, Zhu M, Prinz S. Aconitum intraditional Chinesemedicine-A valuable drug or an unpredictable risk. J Ethnopharmacol. 2009;126(1):18-30.

6. Zhang $\mathrm{H}$, Fan X, Tao J. Exploration on eighteen incompatible medicaments of asthma prescriptions based on association rules. J Nanjing TCM Univ. 2010;26:89-92.

7. Wang Q, Zhong GS, Liu J. Analyzing the Chinese patent medicine containing eighteen incompatible herbs and nineteen herbs of mutual antagonism in the Chinese National Essential Drug List- Chinese patent medicine. China J Tradit Chin Med Pharm. 2011;26:1082-6.

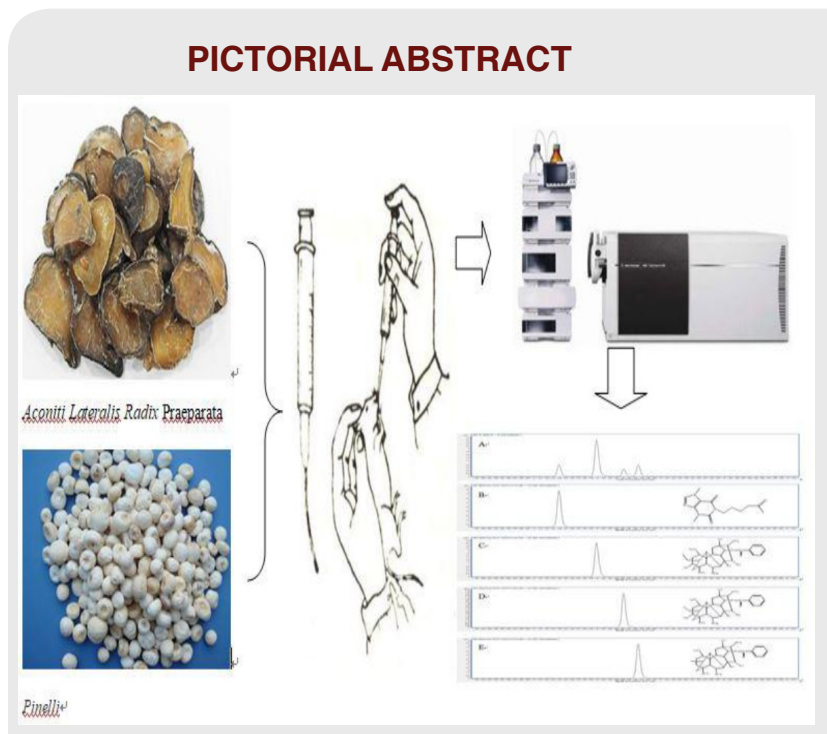

\section{ABOUT AUTHORS}

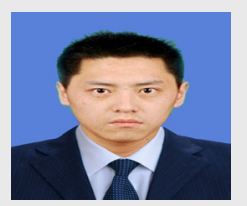

Wu Jian, Assistant professor at Research Center on Life Sciences and Environmental Sciences of Harbin University of Commerce. His area of interest is analytical method development and pharmacokinetic studies.

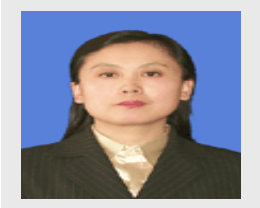

Li Wen-lan is a Professor of Harbin University of Commerce. Areas of interest: Analytical method development and validation.

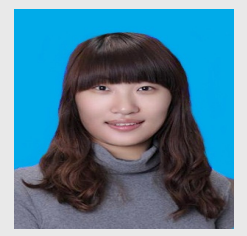

Ying $\mathrm{Xu}$ has completed her M.Sc. and Ph.D. degrees in Natural Products Chemistry from the Shen yang Pharmaceutical University, Shen yang, China, in 2009 and 2012. Her research areas include Natural Products Chemistry, Chinese materia medica, Analytical Chemistry, Pharmaceutical chemistry, Pharmacology.

\section{SUMMARY}

- Incompatible pairs means two herbs are not suitable for clinical application together, otherwise there will be detrimental to therapeutic efficacy, causing toxicity to the body. As recorded in Chinese Pharmacopeia, co-administration of Pinellia (Banxia) and Aconiti lateralis Radix Praeparata (Fuzi) was prohibited. It has always been disputed, because there are many traditional Chinese herbal prescriptions including incompatible pairs, such as Wufu Jingfang, containing Aconiti lateralis Radix Praeparata and Pinellia. In this study, a rapid, sensitive and robust UPLC-MS/MS method for simultaneous determination of benzoylaconine, benzoylmesaconine and benzoylhypaconine in plasma was developed and validated. Pharmacokinetic study was performed to explore the in vivo differences of the three alkaloids after oral administration of FuziBanxia and each single herb extracts. After Banxia in combination with Fuzi, the results showed that faster absorption and higher exposure of BMA and BHA in rat plasma.

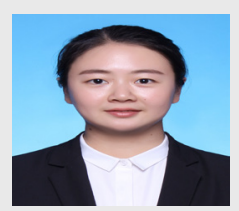

Yu Miao, born in April 1988, working as an assistant researcher, Harbin University of Commerce, majoring in traditional Chinese medicine pharmacology.

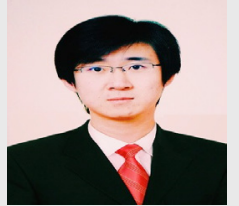

Liu Rijia has done his Ph.D. from Shandong University of Environmental Science and Engineering, Jinan, China in 2014. Area of interest pharmacology, new drug discovery, new drug synthesis, environmental toxicology. He has published 6 papers in the international journal.

Cite this article: Jian WU, Wen-lan LI, Ying XU, Ying-jie L, Ri-jia L, Miao L. Pharmacokinetic Differences of Three Aconitum Alkaloids from Aconiti lateralis Radix Praeparata and Compatibility with Pinellia in Rats. Indian $\mathrm{J}$ of Pharmaceutical Education and Research. 2019;53(2):236-41. 